\title{
TTR
}

Traduction, terminologie, rédaction

\section{De l'Union européenne à la Bretagne : la traduction dans les politiques linguistiques}

\section{David ar Rouz}

Volume 26, numéro 1, 1er semestre 2013

Traduction et contact multilingue

Translation and Multilingual

URI : https://id.erudit.org/iderudit/1036949ar

DOI : https://doi.org/10.7202/1036949ar

Aller au sommaire du numéro

\section{Éditeur(s)}

Association canadienne de traductologie

ISSN

0835-8443 (imprimé)

1708-2188 (numérique)

Découvrir la revue

Citer cet article

ar Rouz, D. (2013). De l'Union européenne à la Bretagne : la traduction dans les politiques linguistiques. TTR, 26(1), 37-72. https://doi.org/10.7202/1036949ar

\section{Résumé de l'article}

Que la Bretagne se soit dotée d'une politique linguistique pourra en surprendre plus d'un. La France n'est-elle pas monolingue ? Quelles langues s'agit-il de traduire dans ce cadre ? Et surtout, pour quoi faire ? Que peut bien apporter l'analyse de la situation bretonne à la compréhension de la politique linguistique de l'Union européenne, l'organisation qui dispose à ce jour des plus grands services de traduction au monde ? Cette comparaison permet pourtant de mieux percevoir le rôle social et la valeur de la traduction professionnelle, non seulement sur le plan économique, mais aussi, et surtout, sur le plan de la communication. Car elle favorise un certain rapport à la langue, et donc à soi-même, dans l'échange et la négociation. La traduction professionnelle prend alors une place prépondérante, voire devient la politique linguistique, et c'est sa quasi-absence dans les écrits sur les politiques linguistiques qui a de quoi interpeller. 


\title{
De l'Union européenne à la Bretagne : la traduction dans les politiques linguistiques
}

\author{
David ar Rouz \\ Université de Haute-Bretagne
}

\begin{abstract}
Résumé
Que la Bretagne se soit dotée d'une politique linguistique pourra en surprendre plus d'un. La France n'est-elle pas monolingue? Quelles langues s'agit-il de traduire dans ce cadre? Et surtout, pour quoi faire? Que peut bien apporter l'analyse de la situation bretonne à la compréhension de la politique linguistique de l'Union européenne, l'organisation qui dispose à ce jour des plus grands services de traduction au monde? Cette comparaison permet pourtant de mieux percevoir le rôle social et la valeur de la traduction professionnelle, non seulement sur le plan économique, mais aussi, et surtout, sur le plan de la communication. Car elle favorise un certain rapport à la langue, et donc à soi-même, dans l'échange et la négociation. La traduction professionnelle prend alors une place prépondérante, voire devient la politique linguistique, et c'est sa quasi-absence dans les écrits sur les politiques linguistiques qui a de quoi interpeller.
\end{abstract}

\begin{abstract}
Many may find it surprising that Brittany has a language policy. Isn't France a monolingual country? What languages could be translated in this context? And, above all, for what purpose? What could the analysis of the situation in Brittany bring to our understanding of the language policy of the European Union, the organisation with the largest translation services in the world? In fact, this comparative analysis enables us to better understand the social role and value of professional translation, not only in the economic field, but also, and especially, in the realm of communication. This is because translation fosters a particular relationship with language, and oneself, through exchange and negotiation. Thus, professional translation is dominant, and even becomes the languague policy itself. It is its near absence in language policy literature that can be called into question.
\end{abstract}

Mots-clés: traduction institutionnelle, politique linguistique, Union européenne, Bretagne, traductologie anthropologique 
Keywords: institutional translation, language policy, European Union, Brittany, anthropological translation studies

Que nous parlions de politique linguistique pour l'Union européenne ne saurait surprendre: avec aujourd'hui vingt-quatre langues officielles, cette organisation supranationale, qui a inscrit le multilinguisme dans ses textes fondateurs (Règlement $n^{\circ} 1$ du Conseil de la CEE, 6 octobre 1958), possède les plus grands services linguistiques du monde. Ces derniers cristallisent en euxmêmes une certaine politique linguistique, suivant la définition qu'en donne Louis-Jean Calvet dans Le marché aux langues (2002, p. 16) d'«ensemble des choix conscients concernant les rapports entre langue(s) et vie sociale». Que nous associions le terme à la région de Bretagne pourra sembler plus incongru. C'est pourtant bien explicitement d'une politique linguistique qu'a souhaité se doter la région (Région Bretagne, 2004) et, en faisant de l'association "Office de la langue bretonne» un établissement public de coopération culturelle en 2010, elle se donne clairement les moyens d'une "planification linguistique», c'est-à-dire d'une mise en œuvre de cette politique.

Pour comprendre ce qui justifie ces actions, nous nous attacherons dans un premier temps à décrire les situations de multilinguisme des deux territoires envisagés ici, à savoir l'Union européenne et la Bretagne. Dans un deuxième temps, nous nous efforcerons de comprendre la place qu'occupe la traduction professionnelle dans leur politique linguistique. Nous verrons ensuite que la traduction n'a pas la place quelle mérite dans les études relevant des politiques linguistiques, sans doute parce qu'on se limite trop souvent à envisager les situations de multilinguisme en termes statistiques. Meylaerts (2009, p. 9-10) a aussi constaté cette lacune, noté quelques rares exceptions, et commencé à la combler, tout en appelant «des recherches ultérieures, davantage comparées».

Nous proposerons donc ici, en comparant les deux territoires, de considérer la valeur de la traduction en termes de rapport entre son coût, maintes fois souligné, et son bénéfice, finalement rarement évoqué, ces deux éléments de l'analyse ne pouvant se limiter à des données purement quantitatives. De cette étude émergera la traduction professionnelle, non seulement comme une 
«branche» de la politique linguistique (Calvet, 2007, p. 55), mais comme une politique linguistique en elle-même.

\section{Le multilinguisme de l'Union européenne}

\subsection{Les langues officielles}

Nous l'avons dit, l'Union européenne (UE), qui regroupe vingthuit États depuis 2010, admet aujourd'hui vingt-quatre langues officielles. À elles seules, ces langues offrent un panorama varié de situations sociolinguistiques: du maltais, unique représentant de la famille afro-asiatique (groupe sémitique, comme l'arabe et l'hébreu), seule langue aussi qu'on trouve dans un unique État, à l'allemand, qui compte le plus de locuteurs dans l'UE et dans le plus d'États différents, en passant par les langues dont la diffusion internationale est bien supérieure, comme l'espagnol, l'anglais, le portugais et le français (si l'on compte les locuteurs dont c'est la langue seconde), ou par une langue telle l'irlandais, que l'on peut considérer comme "minorisée », c'est-à-dire souffrant d' «una clara restricció dels usos en determinats àmbits i en algunes funcions d'ús» (Solé i Camardons, 2001, p. 74¹).

Les données présentées dans le Tableau 1 (page suivante) mettent en évidence le multilinguisme «officiel» de l'UE. Il n'est pas inutile de rappeler en outre que le multilinguisme du territoire envisagé ici ne se limite pas aux langues officielles. Nous laisserons de côté dans le cadre de cet article les langues des migrants, mais nous mentionnerons tout de même une autre catégorie de langues.

\subsection{Les langues européennes non officielles}

Car, si l'Europe est le continent le moins «riche» en langues, on en dénombre tout de même plus de 200, et nous en avons recensé, pour les seuls pays de l'UE, plus de 130. Bien sûr, ce décompte est toujours problématique: sur quel critère peut-on considérer, comme le fait le SIL (Summer Institute of Linguistics), que les sames de Lule, de Pite, de Skolt, d'Ume, du Nord, du Sud, etc., sont des langues différentes, alors que Linguamón ne donne que le same? Le même SIL ne cite aucune des langues d'oil que sont le normand, le poitevin-saintongeais, le gallo, alors qu'il mentionne

1. «Une évidente restriction de son usage dans certains domaines et dans certaines fonctions» (notre traduction du catalan). 
le picard et que toutes apparaissent sur le site de la Délégation générale à la langue française et aux langues de France.

Tableau 1. Langues officielles de l'UE selon le nombre de locuteurs ${ }^{2}$

\begin{tabular}{|l|l|r|r||}
\hline \multicolumn{1}{|c|}{ Langue (FR) } & ISO A3 & Nombre de locuteurs & Proportion UE \\
\hline allemand & DEU & 86191608 & $17,23 \%$ \\
\hline anglais & ENG & 62106000 & $12,41 \%$ \\
\hline français & FRA & 58286200 & $11,65 \%$ \\
\hline italien & ITA & 54006360 & $10,79 \%$ \\
\hline polonais & POL & 38581593 & $7,71 \%$ \\
\hline espagnol, castillan & SPA & 34000000 & $6,80 \%$ \\
\hline néerlandais & NLD & 21020000 & $4,20 \%$ \\
\hline $\begin{array}{l}\text { roumain, daco-rou- } \\
\text { main }\end{array}$ & RON & 20520720 & $4,10 \%$ \\
\hline hongrois & HUN & 12036255 & $2,41 \%$ \\
\hline grec & ELL & 11437240 & $2,29 \%$ \\
\hline portugais & POR & 10510000 & $2,10 \%$ \\
\hline suédois & SWE & 9389961 & $1,88 \%$ \\
\hline tchèque & CES & 9320582 & $1,86 \%$ \\
\hline bulgare & BUL & 6242412 & $1,25 \%$ \\
\hline \hline slovaque & SLK & 5471000 & $1,09 \%$ \\
\hline danois & DAN & 5400000 & $1,08 \%$ \\
\hline finnois, finlandais & FIN & 5005056 & $1,00 \%$ \\
\hline croate & HRV & 4020645 & $0,80 \%$ \\
\hline lituanien & LIT & 3066198 & $0,61 \%$ \\
\hline slovène & SLV & 1882187 & $0,38 \%$ \\
\hline letton & LAV & 1398389 & $0,28 \%$ \\
\hline estonien & EST & 931117 & $0,19 \%$ \\
\hline irlandais & GLE & 859000 & $0,17 \%$ \\
\hline maltais & MLT & 390000 & $0,08 \%$ \\
\hline & & $\mathbf{4 6 2 0 7 2 5 2 3}$ & $\mathbf{9 2 , 3 6 \%}$ \\
\hline
\end{tabular}

2. Nous avons utilisé comme sources pour le nombre de locuteurs soit les chiffres du Summer Institute of Linguistics (SIL International), publiés sur son site http://www.ethnologue.com, soit ceux du site Linguamón - Casa de les llengües (http://www10.gencat.net/casa_llengues/AppJava/fr/index.jsp). On peut bien sûr trouver d'autres sources qui donneront toutes des estimations différentes. Calvet (2002) cite ainsi le Quid et Linguasphere en plus du SIL. 
Ce n'est pas le lieu ici d'entamer la discussion d'une définition de la langue (v. Blanchet, 2000; Ar Rouz, 2010b). Qu'il nous suffise de rappeler l'existence de ces langues non officielles à l'échelle de l'UE, et, pour beaucoup, tout aussi officieuses à l'échelle des États, voire de leurs régions. Rappelons pourtant que certaines d'entre elles viendraient jouer les trouble-fêtes dans le classement établi ci-dessus: le bavarois aurait ainsi plus de locuteurs que le hongrois et le grec; le catalan et le bas-saxon passeraient devant le tchèque et le suédois; s'intercaleraient ensuite le lombard et le napolitain, devant le bulgare, puis le flamand, etc. L'UE ne laisse pas totalement ces langues de côté. Pour preuve, la Commission reconnaît dans sa communication intitulée Multilinguisme: un atout pour l'Europe et un engagement commun l'existence de langues régionales ou minoritaires: "Quelque 60 autres langues font également partie de son patrimoine et sont parlées dans certaines régions ou par des groupes spécifiques » (Commission européenne, 2008c, p. 5). Le Parlement européen indiquait quant à lui en 2003 :

Selon les données de la Commission, on dénombre plus de 60 communautés linguistiques minoritaires ou régionales autochtones dans l'Union européenne et [...] ce chiffre sera plus que doublé à la suite de l'élargissement. (Parlement européen, 2003, p. 375)

Mais on peut constater, d'une part, que le seul organisme que la Commission finançait pour valoriser ces langues, à savoir le Bureau européen des langues moins répandues (EBLUL de son acronyme anglais), a fermé en février 2010, même si la Commission continue de subventionner le réseau Network to Promote Linguistic Diversity (NPLD); d'autre part, l'inclusion effective de ces langues dans la politique linguistique de l'Union dépend de la volonté des États membres où elles sont parlées. Ainsi, les citoyens de langue basque, catalane, écossaise, galicienne, galloise peuvent désormais utiliser leur langue pour s'adresser aux institutions de l'Union en vertu d'un accord avec les gouvernements concernés, qui prennent à leur charge les frais de traduction (Branchadell, 2005 pour les langues espagnoles; European Bureau for LesserUsed Languages, 2009 pour le gallois; Hicks, 2009 pour le gaélique écossais). 


\section{La politique linguistique de l'Union européenne}

Nous avons spontanément associé politique linguistique et traduction, mais il peut être judicieux, avant d'aller plus loin, de nous demander quelle est précisément la politique linguistique de l'Union, tant il est vrai que la traduction peut apparaître comme la grande absente de certains écrits sur les politiques linguistiques. Ainsi, on ne peut quêtre frappé, à la lecture des ouvrages de Louis-Jean Calvet, de ne voir traiter de la traduction qu'à travers le prisme des problèmes de coûts financiers ou de lourdeur administrative (voir en particulier Calvet, 1996, p. 101; Calvet, 2002, p. 43-50, p. 100, p. 135-137 et p. 192-194). Même lorsqu'il évoque les moyens utilisés par les êtres humains pour dépasser leurs différences linguistiques, la traduction n'y figure pas:

Il est rare de rencontrer des situations dans lesquelles se manifestent de façon permanente des problèmes de communication. Lorsqu'ils sont confrontés à de tels problèmes, les êtres humains se débrouillent pour les résoudre d'une façon ou d'une autre, soit en bricolant des «langues approximatives" (les pidgins), soit en apprenant la langue de l'autre et en abandonnant éventuellement la leur, soit enfin en utilisant des langues véhiculaires. (Calvet, 2002, p. 17)

Il faut attendre une conférence de 2003 présentée au Caire et intitulée «De la science en arabe à la traduction: centralité et diversité», au colloque Quand la science parlait arabe, et un article (dans Nowicki et Oustinoff, 2007, p. 45-57), pour voir Calvet faire une place aux traducteurs et interprètes dans son modèle gravitationnel, en tant que liens entre les communautés linguistiques (p.46), et aux politiques de traduction dans les politiques linguistiques (p. 55).

Pourtant, la traduction est au cœur de la politique linguistique de l'UE, comme le montre l'abondance de documents publiés sur la question par la Commission européenne, grâce à sa Direction générale de la Traduction (DGT), le plus grand service de traduction au monde. Calvet nous dirait peut-être que ce n'est pas par nécessité de "communication", mais plutôt pour maintenir "la fonction identitaire ou symbolique» des langues en présence (Calvet, 2002, p. 100). Sans doute. Mais nous allons voir que le régime linguistique de l'Union fonctionne relativement bien 


\section{Langues officielles de l'Union européenne}

Roumain

Bulgare

Irlandais(*)

Tchèque

Slovène

Slovaque

Polonais

Maltais

Lituanien

Letton

Hongrois

Estonien

Suédois

Finnois

Espagnol

Portugais

Grec-

Danois

Anglais

Néerlandais( $\left.{ }^{* *}\right)$

Italien $(* *)$

Français(**)

Allemand $\left.{ }^{* *}\right)$

A

1953

1973

$19811986-1995$

$2004-2007$

(*) Irlandais: langue du traité depuis 1973. Basque, catalan et galicien: statut particulier depuis 2006.

${ }^{* *}$ Ces quatre langues étaient utilisées par la Communauté européenne du charbon et de l'acier, fondée en 1950, qui a marqué la première étape dans la formation de l'Union européenne actuelle. En 1958, les langues officielles ont été l'objet du premier règlement du Conseil, lequel est modifié à chaque nouvelle adhésion.

Figure 1. Langues officielles de l'UE en 2008

(C) Communautés européennes (2008b, p. 3) 
malgré sa grande complexité, et que la réflexion sur sa valeur ne devrait pas se limiter à une notion de coût financier, car, comme l'écrit Michael Cronin (2003, p. 73), «seeing translation as solely a problem is to fail to take account of the potential translation offers for genuine biocultural diversity in the contemporary world $»^{3}$.

\subsection{Que disent les textes fondateurs?}

Les études de la DGT (Vieilledent-Monfort,2009; Pariente,2010) le montrent bien: la traduction est présente dès la fondation des premières Communautés européennes, avec surtout le règlement $\mathrm{n}^{\circ} 1$ de $1958 .{ }^{4}$ Le principe de l'égalité des langues officielles, celles demandées par les États membres à leur entrée, n'a jamais été abandonné, malgré les élargissements successifs qui ont fait passer leur nombre de 4 à 6, 7, 9, 11, puis 20 en 2004, 23 en 2007 et 24 aujourd'hui, une histoire que l'on appréhende facilement dans le graphique présenté à la page précédente (Figure 1).

Catherine Vieilledent-Monfort l'a très bien expliqué lors d'une table ronde (DLF Bruxelles-Europe, 2010): ce régime linguistique «intégral» répond à des principes édictés dans les traités fondateurs de l'Union, et renforcés par le traité de Lisbonne:

[Les] principes de diversité et de non-discrimination, d'une part, de citoyenneté européenne et de démocratie, d'autre part. Le respect de cette diversité et la mise en ouvre des droits linguistiques sont en effet nécessaires pour deux raisons au moins:

- les citoyens et les tribunaux doivent comprendre le droit qui s'applique directement à eux et qu'ils appliquent: c'est l'enjeu de la validité juridique;

- ils doivent participer au processus d'élaboration du droit communautaire: c'est l'enjeu de la légitimité des institutions européennes. (Ar Rouz, 2010a, p. 2)

Elle a mentionné plus spécifiquement l'article 3 du Traité sur l'UE qui consacre la diversité linguistique, la Charte des droits fondamentaux ainsi que l'autre partie du Traité de Lisbonne, le

3. «Voir la traduction uniquement comme un problème revient à passer à côté du potentiel qu'elle offre pour une véritable diversité bioculturelle dans le monde contemporain.» (Notre traduction)

4. On en trouvera une version consolidée dans Vieilledent-Monfort (2009, p. 65). 
Traité sur le fonctionnement de l'UE, dont la deuxième partie est intitulée «Non-discrimination et citoyenneté de l'Union».

Le règlement $\mathrm{n}^{\circ} 1$ de 1958 fixe le régime linguistique de l'Union en en déterminant les langues officielles (article premier), puis ce qu'on appelle le «régime linguistique externe", c'est-à-dire les relations entre les institutions, les États membres et les citoyens relevant de la juridiction de ces États (articles 2 et 3). Il stipule ensuite que «les règlements et les autres textes de portée générale», ainsi que le Journal officiel de l'UE doivent être publiés dans les langues officielles. Enfin, il précise que les institutions «peuvent déterminer les modalités d'application de ce régime linguistique dans leurs règlements intérieurs» (article 6).

En termes de politique linguistique, de «choix conscients concernant les rapports entre langue(s) et vie sociale» (Calvet, 2002, p. 16) pour reprendre la définition citée en introduction, il ressort donc de ce règlement que les institutions doivent s'organiser pour respecter l'égalité de toutes les langues officielles au regard des États membres et des citoyens. Cela implique que tous peuvent s'adresser aux institutions dans une des langues officielles, auxquelles il faut ajouter les langues pour lesquelles ont été conclus des accords spécifiques (langues d'Espagne et de GrandeBretagne), que les institutions sont tenues de leur répondre dans cette langue et de s'adresser aux États membres dans leur langue officielle, de publier les textes juridiquement contraignants ou de portée générale dans toutes les langues officielles simultanément.

On retient également que le régime interne des institutions est défini par chacune d'elles. En pratique, pour ne donner que ces exemples, la Commission européenne fonctionne, "pour des raisons d'efficacité» (Vieilledent-Monfort, 2009, p. 14), avec trois langues procédurales, l'anglais, le français et l'allemand, tandis que la Cour de Justice délibère en français.

Le Parlement européen, lui, a «l'obligation [...] d'assurer le multilinguisme le plus large possible» (site Internet du Parlement). Outre la mise à disposition de la législation à tous les citoyens de l'Union, il s'agit en effet de permettre aux élus de participer aux débats, puisque tout citoyen a le droit d'être élu député européen, indépendamment de ses compétences en langues étrangères. Le Parlement fixe donc des règles strictes de l'usage des langues dans 
l'article 146 de son règlement (Règlement du Parlement européen) et dans le Code de conduite du multilinguisme établi en 2006 et revu en 2008 (Parlement européen, 2008). Ce dernier doit permettre un "multilinguisme intégral maîtrisé » par une approche pragmatique impliquant notamment l'utilisation de langues pivots ou une plus grande souplesse dans les phases préparatoires des documents.

Il est par conséquent évident que le régime externe exigera des services de traduction importants dans toutes les langues officielles. Il semble très clair aussi que la traduction professionnelle intervient nécessairement à un stade ou un autre dans le fonctionnement interne des institutions, mais les besoins de chacune en la matière seront différents selon son régime linguistique interne et son rôle dans la machine communautaire.

\subsection{Les services de traduction de l'Union européenne}

Dès 1986, le service de traduction de la Commission européenne $(\mathrm{SdT})$ fait figure de "plus grand service de traduction du monde» (Vieilledent-Monfort, 2009, p. 33). C'est toujours le cas aujourd'hui avec, en plus, l'idée qu'il est aussi l'un des meilleurs, comme l'écrit l'ancien Directeur général, Karl-Johan Lönnroth (ibid., p. 6). Le service est devenu en 2003 une direction générale à part entière, la Direction générale de la Traduction (DGT), et, étant donné le rôle important de la Commission européenne dans la production des propositions législatives et dans les relations aussi bien avec les autres institutions qu'avec l'extérieur de l'Union, la DGT a tendance à masquer les nombreux services linguistiques que compte l'UE.

Pourtant, c'est aussi sous son impulsion qu'ils acquièrent ensemble une nouvelle visibilité. On trouve ainsi dans Pariente (2010, p. 49) le graphique de la page suivante (Figure 2), qui rend compte des différents services, avec leurs effectifs incluant aussi bien les traducteurs et interprètes que le personnel d'encadrement et les assistants. À noter que la DGT ne compte que des traducteurs, les interprètes de la Commission étant rassemblés dans la DG Interprétation qui, étrangement, n'apparaît pas dans ce schéma malgré ses quelque 500 interprètes.

On peut dans certains cas préciser les estimations des effectifs: sont ainsi mentionnés sur le site de la Cour de justice des effectifs de 876 traducteurs et 70 interprètes, de plus de 700 traducteurs et 
430 interprètes sur celui du Parlement. Dans ces deux institutions, les deux activités sont d'ailleurs confiées à des directions générales distinctes, alors que le Conseil, par exemple, fait appel aux interprètes du Parlement et que les institutions disposant de plus petits services les nomment plutôt "services linguistiques». Ajoutons qu'il s'agit là des effectifs de fonctionnaires, qui ne tiennent donc pas compte du personnel extérieur. Le Parlement indique notamment compter sur une réserve de plus de 2500 interprètes externes. Quant à la DGT de la Commission, on trouvera le détail de son personnel dans Pariente (2010, p. 60) et son organigramme détaillé dans Vieilledent-Monfort (2009, p. 114).

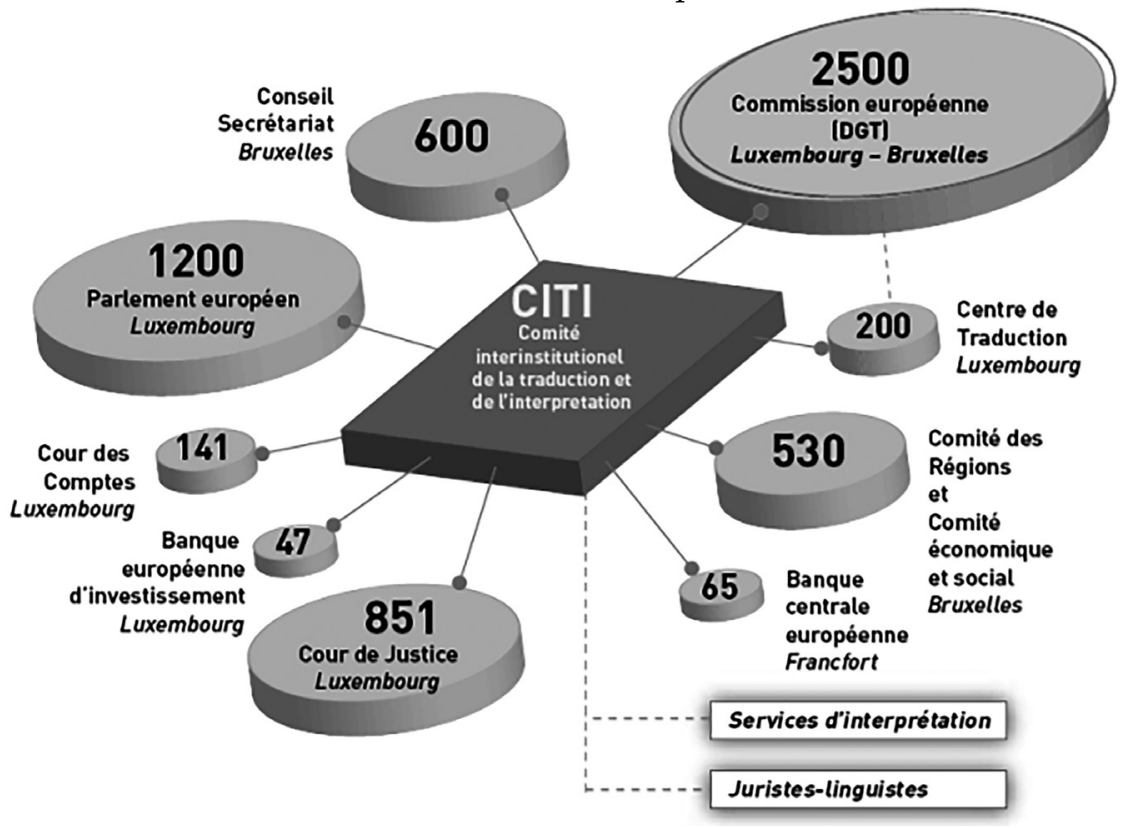

Figure 2. Collaboration institutionnelle en traduction et interprétation (C) Union européenne (Pariente, 2010, p. 49)

Enfin, nous avons aussi essayé de recueillir le nombre de pages (normées) traduites par an dans chaque service et nous obtenons le graphique ci-après (Figure 3), où DGTE désigne la Direction générale de la traduction et de l'édition du Parlement européen, DG A III, la Direction A III (traduction et production 
des documents) du Conseil de l'UE, CdT correspond au Centre de traduction auquel ont recours les différentes agences européennes, et DGT CJCE est la Direction générale de la Traduction de la Cour de justice des Communautés européennes. Les sources sont Vieilledent-Monfort (2009, p. 102) et les sites des autres institutions.

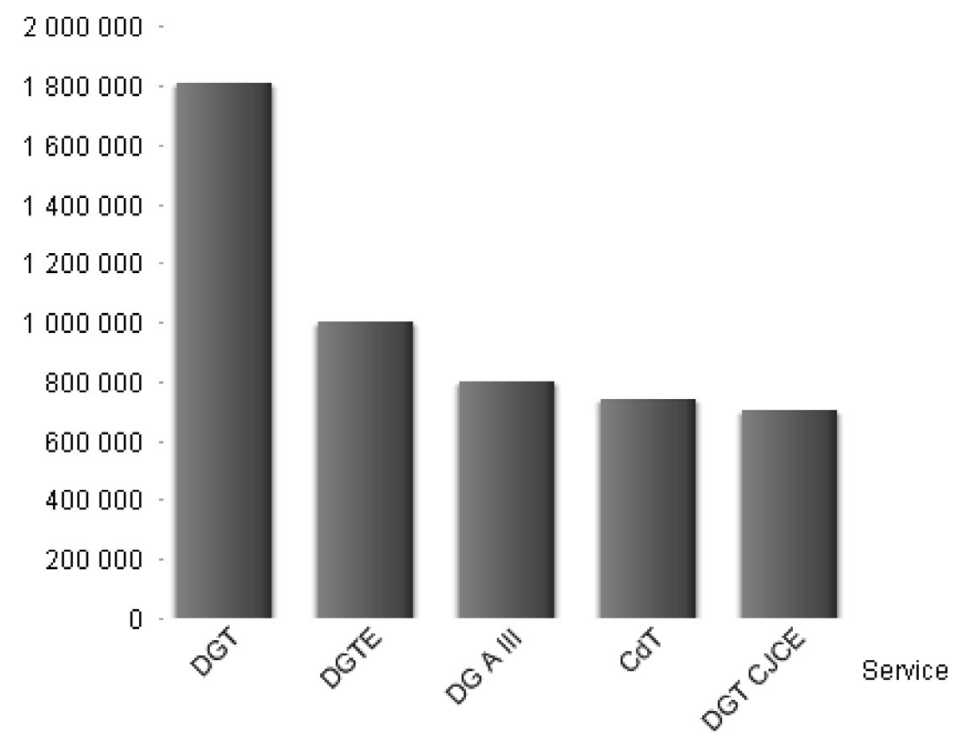

Figure 3. Production de services de traduction de l'UE

(C) David ar Rouz, 2011

On mesure l'importance de ces services pour l'UE, que ce soit sur le plan de la production, sur le plan institutionnel (ils ont désormais une Direction générale à part entière dans beaucoup d'institutions) ou sur le plan des effectifs. Le graphique de la page suivante (Figure 4) montre la proportion que représentent les traducteurs et interprètes dans les effectifs des institutions, calculés à partir des sites des institutions ou de Rinsche et al. (2009, p. 36), Pariente (2010, p. 49) et du tableau des effectifs des institutions (Parlement européen). Nous n'avons pu toutefois calculer le pourcentage que représente le personnel du Centre de Traduction par rapport à 
celui des agences européennes pour lesquelles il travaille, car les effectifs de ces dernières sont difficiles à trouver et plus variables que ceux des autres institutions.

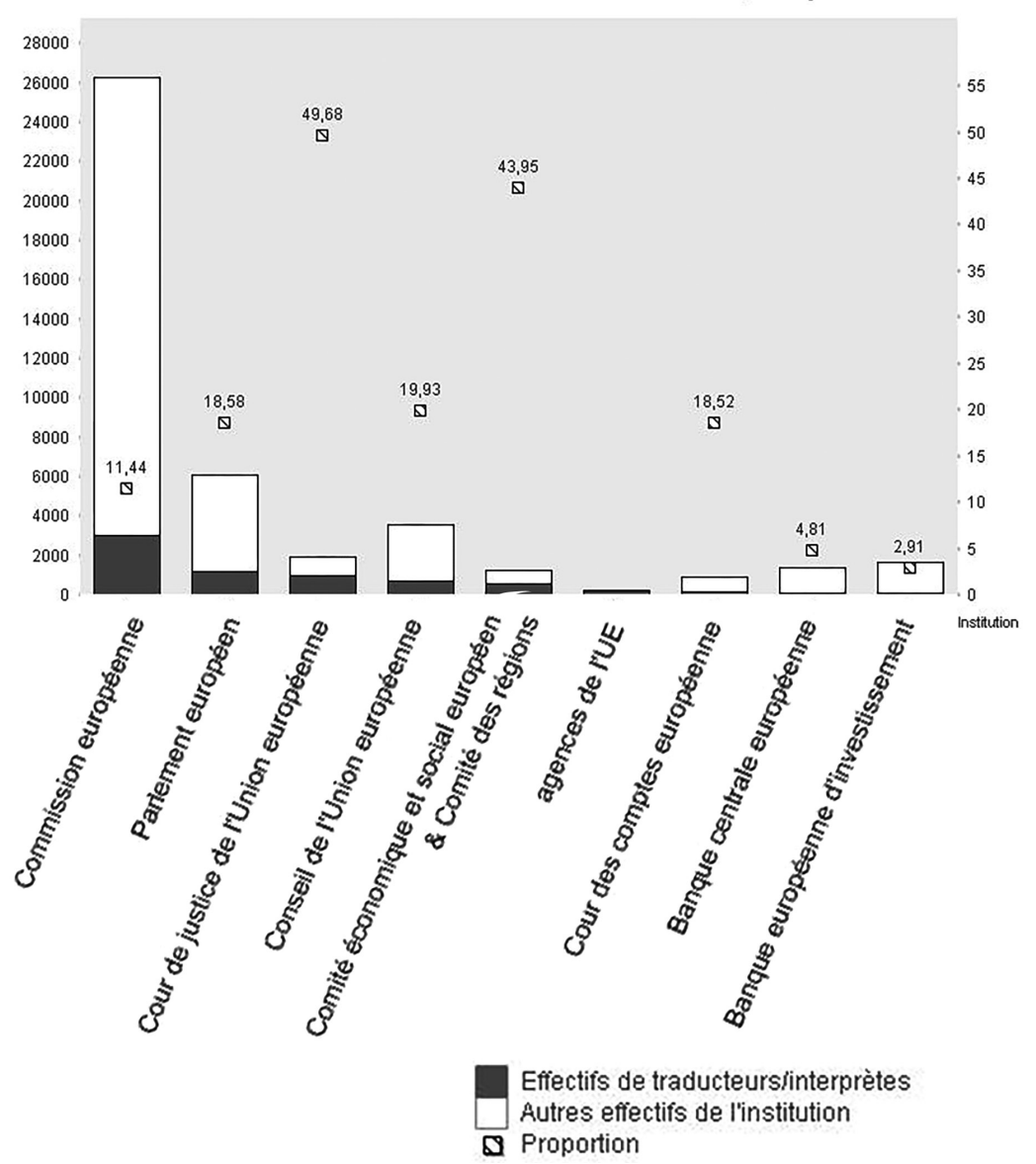

Figure 4. Effectifs des services de traduction/interprétation de l'UE

(C) David ar Rouz, 2011

On voit ici que la DGT et la DGI, les plus grands services de traduction et d'interprétation en chiffres absolus, ne sont pas, proportionnellement à l'effectif total de la Commission européenne, les plus importants. Ceux de la Cour de Justice ou le service commun au Comité économique et social européen et au Comité des régions 
se trouvent loin devant, suivis par ceux du Conseil, du Parlement et de la Cour des comptes. Constater l'existence et l'importance de ces services à partir de chiffres ne suffit pas, toutefois, à rendre compte de leur valeur. Pour comprendre cette valeur, il nous faudra nous interroger sur leur rôle. Auparavant, nous allons présenter la situation de la région de Bretagne, qui nous offrira un point de comparaison pour les analyses que nous souhaitons mener.

\section{Le multilinguisme et la politique linguistique de la Bretagne}

\subsection{Sociolinguistique bretonne}

La carte ci-dessous (Figure 5) donne d'emblée une idée assez précise de la situation sociolinguistique de la Bretagne, puisqu'elle indique l'évolution d'une frontière linguistique du IX ${ }^{\mathrm{e}}$ siècle (la ligne la plus à l'est) à aujourd'hui.

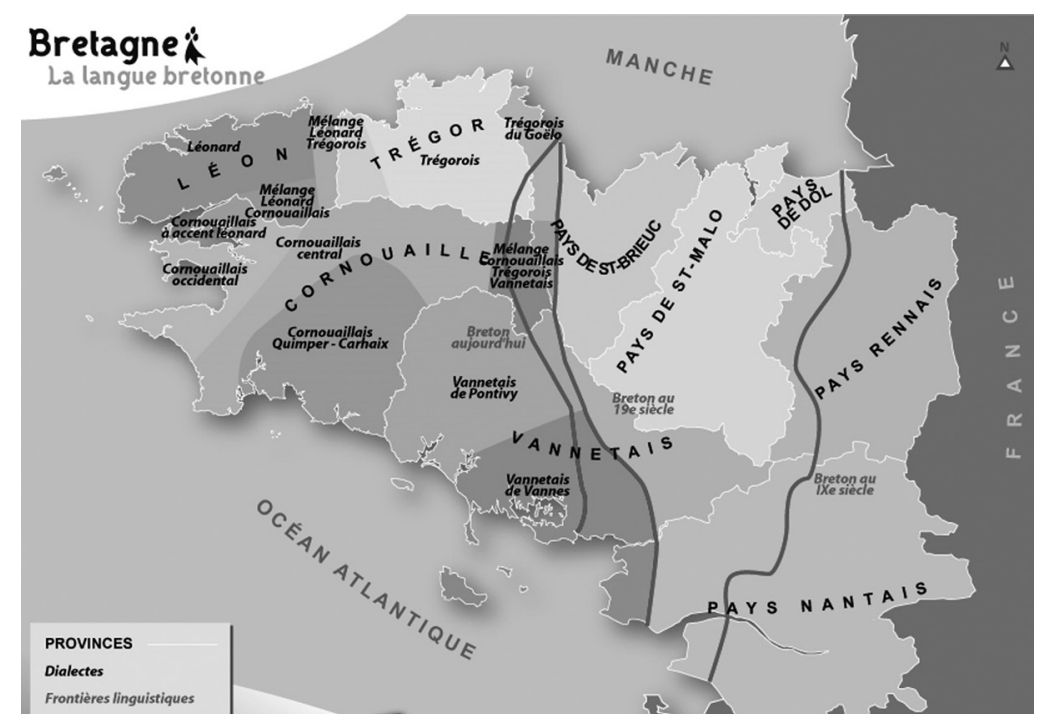

Figure 5. Bretagne, la langue bretonne

(C) Mikael Bodlore-Penlaez (2008, geobreizh.com)

Bien entendu, étant donné la grande mobilité des populations de nos jours, on ne peut considérer cette frontière comme étanche, et les bretonnants vivent aussi aujourd'hui dans les grandes villes que 
sont Nantes et Rennes et dans leur périphérie. C'est ainsi qu'on peut contraster cette carte avec la suivante (Figure 6), produite par l'Office de la langue bretonne (2006, p. 6):

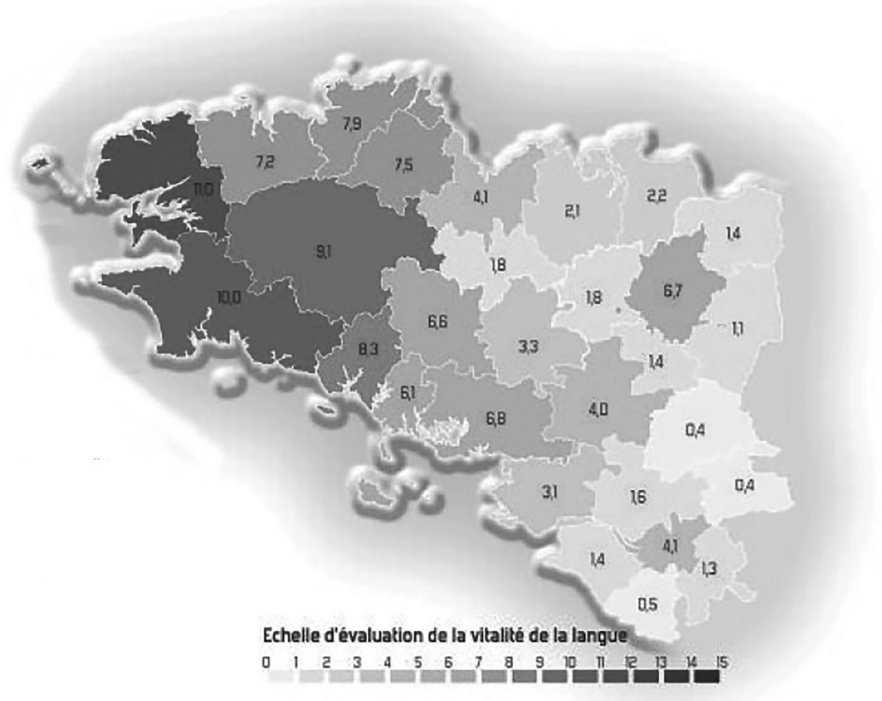

Figure 6. Vitalité de la langue bretonne

(C) Office de la langue bretonne (2006)

La limite de la première carte (Figure 5) montre du moins l'existence de deux langues « régionales », le breton à l'Ouest, le gallo à l'Est, en plus du français aujourd'hui dominant partout. Après un fort déclin tout au long du XX ${ }^{\mathrm{e}}$ siècle, la première, langue indoeuropéenne de la branche celtique brittonique, apparentée par conséquent au gallois et au cornique, est encore parlée de nos jours par environ 206000 personnes, dont 172000 en Basse-Bretagne, c'est-à-dire sur le territoire historique de la langue, et environ 12000 enfants scolarisés dans les filières bilingues, Div Yezh et Dihun, ou immersive, Diwan (Gaillard, 2009, à l'occasion de la parution du dernier sondage TMO analysé par Fañch Broudic dans Parler breton au XXI siècle, Brest: Emgleo Breiz, 2009).

Le gallo, langue d'oil tout comme le français, est présent en Haute-Bretagne, mais il est beaucoup plus difficile d'en estimer le nombre de locuteurs, car beaucoup considèrent qu'il s'agit d'un 
«patois», de «français déformé». La preuve en est que l'INSEE a dénombré 28300 locuteurs actifs en 1999 (Le Boëtté, 2003,p. 18), alors que le Centre de Recherche sur la Diversité Linguistique de la Francophonie (CREDILIF), de l'Université de Rennes 2, estime leur nombre entre 200000 et 400000 (Chevalier, 2008).

Ce trop rapide tableau brossé, il nous intéresse maintenant de constater qu'aujourd'hui, ces deux langues sont reconnues par le Conseil régional de Bretagne et font donc l'objet d'une politique linguistique déclarée (Région Bretagne, 2004). Cela va à l'encontre de l'affirmation de Calvet selon laquelle:

Seul l'État a le pouvoir et les moyens de passer au stade de la planification, de mettre en pratique ses choix politiques. C'est pourquoi, sans exclure la possibilité de politiques linguistiques qui transcendent les frontières (c'est par exemple le cas de la francophonie) ni celle de politique linguistique concernant des entités plus petites que l'État (sur les langues régionales par exemple), il faut admettre que, dans la plupart des cas, les politiques linguistiques sont le fait de l'État ou d'une entité disposant au sein de l'État d'une certaine autonomie politique (comme la Catalogne, la Galice ou le Pays basque en Espagne). (Calvet, 1996, p. 10-11)

La Bretagne ne jouit certes pas de la même autonomie que les communautés autonomes espagnoles, son budget ne soutient aucune comparaison avec bon nombre d'autres régions européennes, mais le plus frappant est que cette politique linguistique à l'échelle régionale intervient dans un État, la France, dont l'image est plutôt celle d'un bloc monolingue.

Il ne nous appartient pas d'analyser dans le détail toutes les actions définies par cette politique linguistique. Les efforts portent en grande partie sur l'enseignement de ces langues afin de contrebalancer la diminution continue du nombre de leurs locuteurs. Nous nous attacherons ici au volet «traduction» de cette politique.

\subsection{Traduction dans les langues régionales de Bretagne}

La traduction en gallo existe: on peut trouver, à côté de créations récentes, certains ouvrages traduits dans cette langue, notamment 
plusieurs albums de Tintin et un d'Astérix ${ }^{5}$. On ne peut en faire aucune analyse statistique du fait que la langue n'est pas recensée par l'UNESCO dans son Index Translationum, qui rassemble des données concernant les traductions publiées dans le monde. On trouve aussi des ouvrages lexicographiques et toponymiques. La production la plus emblématique d'un nouveau type de traduction dans cette langue est sans doute la traduction des panneaux d'affichage de la station Charles de Gaulle du métro de Rennes (v. Ar Rouz et Le Squère, 2005). En tout état de cause, ces productions sont trop peu nombreuses pour faire exister un service professionnel de traduction en gallo et à l'inverse, en l'absence d'un tel service, elles restent rares.

Pour le breton, les deux prestations de traduction écrite et d'interprétation existent. On peut recenser pour la première dans l'Index Translationum 197 traductions du breton entre 1979 et 2005 et 303 traductions vers le breton de 1977 à 2006. Il est intéressant de remarquer, au regard de la situation de domination du français en Bretagne, que la majorité de ces publications sont des traductions du français ou vers le français, comme le montrent clairement les deux graphiques présentés à la page suivante (Figures 7 et 8). Viennent ensuite, comme langues cibles, l'allemand, l'anglais, le gallois et l'occitan et, comme langues sources, l'anglais, le catalan, l'allemand et l'espagnol.

Les ouvrages toponymiques, lexicographiques, voire, plus récemment, proprement terminologiques sont nombreux et cette évolution de la lexicographie à l'étude terminographique systématique de certains domaines correspond aussi à la montée en puissance de la traduction que l'on dirait «technique» depuis 1999. C'est en effet cette année-là qu'a été créé l'Office de la langue bretonne à l'initiative de la région, sous forme d'association financée par différentes collectivités régionales et étatiques, avec dès le départ cinq services, dont le service de Traduction - Conseil (quatre salariés, plus deux réviseurs à temps partiel) et le service de terminologie TermBret (un salarié). Cette association est

5. On trouve chez Rue des Scribes Éditions, à Rennes, les albums d'Hergé suivants: Sus l'ile naire (1993), Les Dorures à la Castafiore (1997), La Cutrie dla Licône (2001) et La Guénochée à Rackham Le Rouge (2005); aux Éditions AlbertRené: Uderzo et Goscinny, Astérix à l'école d'retour, Paris, 2004. 
devenue Établissement public de coopération culturelle, sous le nom d'Office public de la langue bretonne, le 17 septembre 2010 (Région Bretagne, 2010).

\section{LS: BRE}
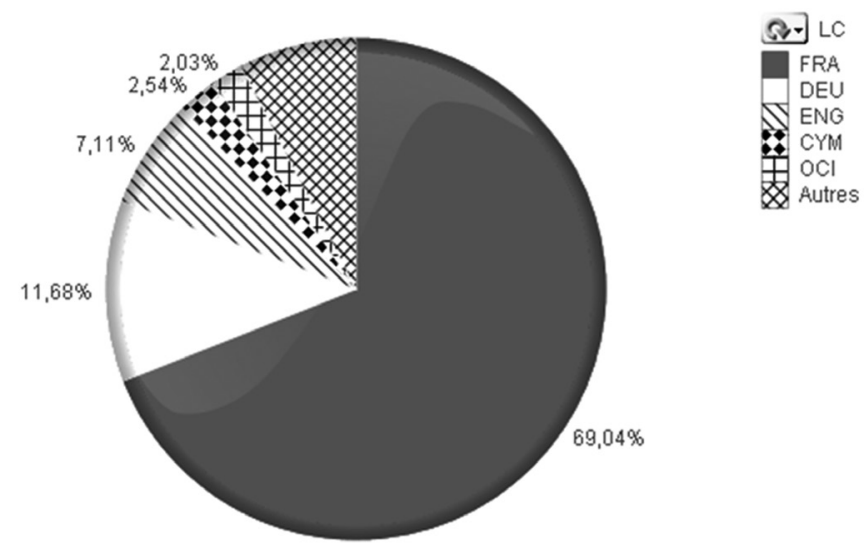

Figure 7. Principales langues cibles des traductions du breton dans l'édition

(C) David ar Rouz (2011)

LC: BRE

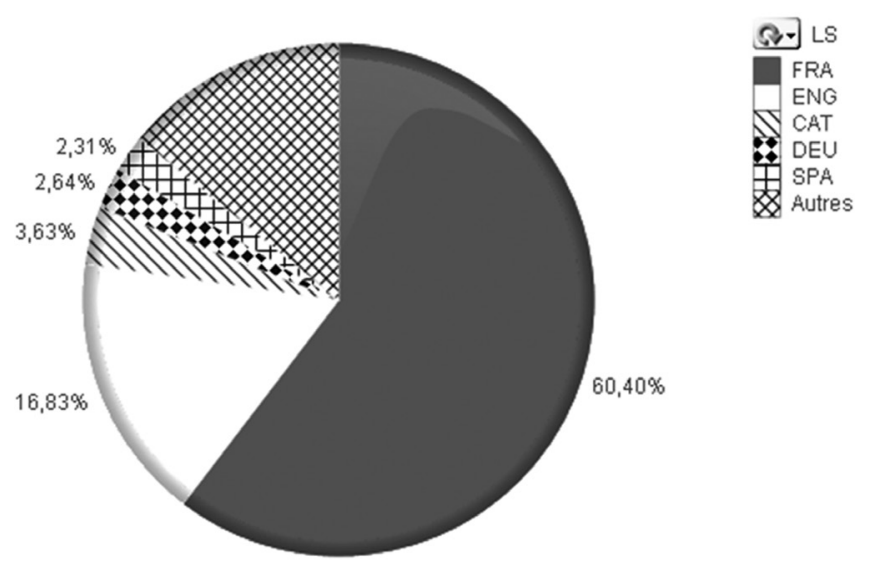

Figure 8. Principales langues sources des traductions vers le breton dans l'édition

(C) David ar Rouz (2011) 


\section{Caractérisation du service de traduction}

Nous avons pris soin de parler ici de «traduction professionnelle». On aura compris, au regard de notre exposé de tels services au sein des institutions européennes, que nous y incluons l'interprétation. Car, socialement parlant, on peut estimer qu'ils sont de même nature. Nous allons plus loin: sur un plan sociolinguistique, chaque individu s'approprie socialement la capacité de langage, ce qui fait qu'il partage «une langue» avec un certain nombre d'autres individus, mais que sa façon d'utiliser ces ressources linguistiques n'est jamais parfaitement superposable à celle d'un autre, en raison de son «histoire de rapports interlocutifs » propre (Peeters, 1999, p. 192). C'est pourquoi il est important de distinguer le moment où le degré d'«étrangeté» de la langue de l'autre justifie le recours à un tiers dont la responsabilité sera de permettre malgré tout l'échange.

Peeters (1999, p. 223-227 et p. 259) explique que cela est rendu possible par la capacité du traducteur à entrer dans deux interlocutions distinctes, avec l'auteur d'une part, avec le bénéficiaire d'autre part, chacune s'effectuant dans les langues alors réputées étrangères. Il faut entendre ici «traducteur» comme la structuration abstraite de relations d'offres et de demandes, c'est-à-dire que nous ne désignons pas par là un individu, mais un acteur, susceptible d'inclure plusieurs individus physiquement distincts et remplissant des fonctions complémentaires, dans le cadre d'un service donné. Cette structuration prend des formes très diverses et variables, qui constituent tout ce que l'on peut en observer, mais distinguent nettement la traduction professionnelle de la conversation par l'intervention d'un tiers redevable aux parties demandeuses du service. La prestation de traduction ainsi définie, il nous faut encore chercher à saisir quelle est sa fonction sociale.

\subsection{Son rôle}

Dans le cadre de l'UE, le rôle que remplissent les différents services de traduction semble assez bien défini, quoiqu'il varie évidemment suivant l'institution, les documents, les situations, les langues. Ainsi, on peut considérer qu'à la DGT, le rôle de toutes les unités est de permettre les interlocutions au sein des institutions européennes, mais aussi (et peut-être surtout) entre 
les institutions européennes et les administrations, les entreprises et les citoyens des États membres. Le rôle des unités anglaises, françaises et allemandes, c'est-à-dire les unités chargées des langues procédurales définies par le régime linguistique interne de la Commission, sera en outre de permettre le fonctionnement de la Commission européenne et la participation de tous les membres du Collège des commissaires à l'élaboration des documents, avant qu'ils ne soient communiqués à l'extérieur de l'institution. Ce sont d'ailleurs les «besoins minimums» des membres du Collège qui déterminent les conditions des traductions intermédiaires et de l'interprétation (Ar Rouz, 2010a).

$\mathrm{Au}$ Parlement, le rôle des DGTE (Direction générale de la traduction et de l'édition) et DGIC (Direction générale de l'interprétation et des conférences) est également de permettre l'interlocution entre l'institution et l'extérieur, ainsi qu'entre les représentants des citoyens réunis lors des sessions parlementaires. Indépendamment des compétences linguistiques des députés, il s'agit en outre dans ces séances plénières qu'ils puissent s'exprimer dans la langue (ou une des langues) de l'État membre dont ils sont l'un des représentants. Ces compétences individuelles entrent en revanche en ligne de compte pour déterminer les besoins en traduction et interprétation dans les commissions, c'est-àdire dans le fonctionnement interne de l'institution (Parlement européen, 2008).

Le rôle que jouent les services de traduction dans les autres institutions pourrait aussi être intéressant à regarder de près, notamment à la Cour de justice, mais la place manquerait de toute façon ici pour mener à bien cette analyse exhaustive. On peut en tout cas constater qu'il y a lieu de distinguer deux rôles légèrement différents :

- permettre l'interlocution entre des personnes (physiques ou morales) qui ne parlent pas la même langue;

- permettre à ces personnes de s'exprimer dans la langue officielle (ou à statut particulier) de leur choix.

Ces deux rôles se recouvrent partiellement. En effet, une personne qui ne possède pas de compétences dans une autre langue que sa langue maternelle n'aura pas le choix de la langue dans laquelle 
elle s'exprime au sein des institutions ou avec elles et la traduction professionnelle jouera alors pleinement ces deux rôles à la fois. En revanche, on peut aussi envisager le cas des députés européens qui auraient les compétences nécessaires pour s'exprimer dans la langue d'autres interlocuteurs plutôt que dans la leur; en tant que représentant des citoyens de tel ou tel État membre, ils préféreront sans doute pourtant en choisir la langue officielle et la traduction le leur permet.

Ont-ils le choix de cette «loyauté» linguistique? C'est une autre question. Le tollé qu'avait provoqué en son temps le discours en anglais de Jean-Claude Trichet à sa prise de fonction comme président de la Banque centrale européenne en 2003 nous autorise à en douter (v. le site Avenir de la langue française). Le phénomène exprime certes des luttes de pouvoir et peut donc à ce titre s'analyser sur le plan social. L'exemple donné par Cronin est à cet égard très révélateur:

The use of an interpreter by the Irish Gaelic leader Hugh O'Neill in his dealings with Elizabeth I (even though he himself spoke English) was a way of initiating dialogue that nonetheless marked the cultural and political distance and difference between the two parties, thereby constructing interpreting as an activity of both interaction and resistance. (Cronin, 2006, p. 87)

Mais il relève peut-être surtout d'une analyse de la valeur, en l'occurrence celle de l'expression dans une langue plutôt que dans une autre. Nous y reviendrons donc dans notre dernière partie.

Si nous nous contentions ici d'affirmer que la traduction permet la communication, nous en resterions au stade du poncif. Examinons donc maintenant la traduction en Bretagne, afin d'approfondir cette notion de rôle de la traduction professionnelle. Ce service pose en effet le problème de manière particulière puisqu'il ne saurait être question dans ce cas d'interlocution, d'intercompréhension au sens où il permettrait

6. «Le recours à un interprète par le chef gaélique irlandais Hugh O’Neill dans ses relations avec Élisabeth $1^{\text {re }}$ (bien qu'il parlât lui-même anglais) était une manière d'ouvrir le dialogue tout en marquant la distance et la différence politiques et culturelles entre les deux parties, faisant ainsi de l'interprétation une activité à la fois d'interaction et de résistance.» (Notre traduction) 
un échange autrement impossible entre des interlocuteurs dont la différence linguistique serait trop grande. Tous les locuteurs du breton ou du gallo aujourd'hui parlent en effet français et la traduction n'a pas pour objectif principal de leur rendre possible une intercompréhension.

Lorsqu'on a affaire à de l'interprétation, elle a le plus souvent lieu du breton au français et vise alors à permettre aux bretonnants de s'exprimer en breton, même en présence de personnes qui ne le comprennent pas. Cela rejoint les cas que nous venons d'évoquer. La traduction écrite a aussi lieu dans ce sens, comme nous l'avons signalé, a priori essentiellement dans le domaine de l'édition. En l'absence de plus d'indications sur les ouvrages traduits, on peut formuler l'hypothèse que ces traductions ont éventuellement un objectif pédagogique, par exemple, dans la publication d'ouvrages bilingues, ou quelles visent à partager et à faire connaître une littérature et une culture originales en les rendant accessibles dans d'autres langues.

Comme l'indiquaient déjà les chiffres présentés dans la partie précédente, le «ratio traducteur» du breton atteint cependant 1,54 , ce qui signifie que l'on traduit davantage vers le breton que du breton. À titre de comparaison, le ratio traducteur du français est presque à l'équilibre, à 1,07, celui du catalan est de 2,32, tandis que l'anglais se situe à 0,11 . Mais ces chiffres ne tiennent compte que des traductions publiées. Or, une grande partie des traductions vers le breton effectuées depuis plus de dix ans, en particulier par l'Office de la langue bretonne, n'entre pas dans cette catégorie et, comme ce type de production est essentiellement unidirectionnel, à partir du français, on peut estimer que le ratio est bien supérieur.

Nous avons expliqué en détail ailleurs (Ar Rouz et Le Squère, 2005, p. 195-203) quelles pouvaient être les motivations des acteurs de cette traduction professionnelle visant le plus souvent un affichage public. S'il n'y est pas question d'intercompréhension, la communication est par contre bien présente à l'esprit de tous, donneurs d'ouvrage comme prestataires. Il s'agit souvent pour les premiers de communiquer une appartenance territoriale, culturelle, historique à des «usitaires » (ibid., p. 196), c'est-à-dire à un public de bretonnants qui pourra faire usage de l'information transmise par la traduction aussi bien qu'à un public de nonbretonnants qui percevra de manières diverses la signification de 
l'action, sa valeur (de l'authenticité au passéisme, en passant par la confusion), à défaut de pouvoir saisir le sens des textes. Ils peuvent aussi souhaiter préserver ainsi un "patrimoine» ou créer, renforcer $\mathrm{du}$ lien social. Certains y voient en outre un atout économique, puisque le symbole linguistique fonctionne parfois comme un label de qualité sur les produits.

Chez les prestataires, on constate que la mise en avant de la différence linguistique pour qu'elle soit reconnue de l'extérieur fait partie des motivations importantes et que l'affichage de traductions dans les langues régionales peut participer à la réappropriation des territoires qui ne se distinguent plus par la pratique sociale de ces langues. L'affichage public des langues dominées est également envisagé comme un moyen de les revaloriser aux yeux des locuteurs qui les ont abandonnées, ne les ont pas transmises ou seraient prêts à les (ré)apprendre.

Dans les deux cas, on voit que la traduction joue bien sûr un rôle social, elle répond à une véritable demande, mais l'échange porte bien plus sur des valeurs que sur des contenus informatifs. Or, c'est justement sur des valeurs qu'a porté la présentation de Catherine Vieilledent-Monfort citée plus haut (DLF BruxellesEurope, 2010). Autrement dit, la valeur de la traduction professionnelle pourrait être liée aussi bien aux valeurs qu'elle permet d'exprimer dans l'échange social (authenticité, qualité, différence, assurance de l'interlocuteur, etc.) qu'à la facilitation de l'échange lui-même.

\subsection{Sa valeur}

Nous proposons d'analyser la valeur de la traduction professionnelle comme un rapport coût/bénéfice. Dans le cas qui nous occupe, on voit bien que le «coût» de la traduction vient imposer des limites au bénéfice que constitue le résultat de l'opération. Dans l'UE, c'est le rôle de la Cour des comptes que d'analyser les coûts et elle l'a fait pour la traduction en 2006 (Cour des comptes, 2006). Ce rapport, tout en reconnaissant les progrès de chaque institution, faisait des recommandations pour éventuellement diminuer les coûts, mais surtout améliorer les prestations, ce qui est une autre façon d'optimiser le rapport coût/bénéfice. 
Dans la notion de «coût de la traduction », on trouve souvent, en ce qui concerne les institutions européennes, deux aspects principaux: la lourdeur administrative, d'une part, et surtout, d'autre part, le coût financier. Calvet écrit par exemple:

Il est une façon de dissimuler cette situation, qui consiste à dire que le coût des services linguistiques des institutions européennes (environ 3000 emplois de traducteurs et 951 emplois d'interprètes) n'est que de deux euros par an et par citoyen européen, ou ne représente que $0,8 \%$ du budget total (Doerflinger, 2001, p. 137). Mais c'est faire l'impasse sur la réalité: la majorité des fonctionnaires européens travaille dans les services linguistiques, même si $0,8 \%$ du budget total peuvent paraître moins importants que $40 \%$ du budget de fonctionnement... Le coût n'est d'ailleurs pas seulement financier. D'une part, il est extrêmement difficile de trouver les interprètes nécessaires pour certaines langues peu parlées. Ainsi, en 2001, aucun candidat ne s'est présenté à un concours de recrutement d'interprètes finnois-grec. On a donc recours à ce qu'on appelle des langues pivots: on traduit par exemple du grec vers l'anglais ou le français, puis de l'anglais ou du français vers le finnois, avec les déperditions sémantiques que le lecteur peut imaginer. D'autre part, le nombre de textes écrits à traduire augmente régulièrement, et la lourdeur du système finira par paralyser l'institution. Le régime linguistique de la Communauté tient donc de la folie. (Calvet, 2002, p. 44)

On retiendra aussi un troisième aspect du coût déploré par Calvet: les «déperditions sémantiques», l'éternelle «trahison» de la traduction. Nous nous contenterons sur ce point de poser la question suivante: les déperditions que l'on peut constater dans le cadre d'une traduction professionnelle sont-elles plus dommageables à l'échange que celles qui surviennent dans n'importe quelle conversation? Un raisonnement simpliste mènerait à la conclusion qu'elles sont multipliées par deux, car il faut deux interlocutions pour que l'échange ait lieu, voire trois si on utilise une langue pivot, mais ce serait oublier les améliorations que la traduction apporte bien souvent à la clarté du message d'origine.

Les données que nous avons fournies précédemment indiquent que les chiffres cités par Calvet en 2002 ne sont plus d'actualité et que l'on est aujourd'hui, après les derniers 
élargissements, bien au-delà de 3951 emplois de traducteurs et d'interprètes. Le contenu de la Figure 4 (v. p. 49) contredit en outre l'affirmation que ces emplois sont majoritaires dans la fonction publique européenne. Concernant le coût financier, en revanche, il semblerait que l'estimation n'ait pas changé en 2010:

Le second mythe est qu'une énorme part du budget de l'Union européenne est consacrée au multilinguisme. Or, les coûts pour la traduction et l'interprétation de toutes les institutions communautaires s'élèvent à moins de $1 \%$ du budget global annuel de l'Union européenne (ce qui correspond à environ $2 €$ par citoyen). À la Commission, les coûts annuels pour la traduction sont estimés à 300 millions d'euros, ce qui équivaut à environ $0,60 €$ par citoyen et par an, montant modeste pour garantir la démocratie et l'égalité de droits entre les citoyens. (Pariente, 2010, p. 56)

Tous les éléments du coût ainsi mis en évidence sont importants à prendre en compte si l'on cherche à déterminer la valeur de la traduction professionnelle dans l'UE. Mais encore faut-il mettre en regard tous les bénéfices qu'elle apporte. Car il ne s'agit pas ici de raisonner uniquement en termes de «grandeurs monétaires ou marchandes» (Grin, 2004, p. 2). Pour autant, il est intéressant de ne pas totalement écarter cet aspect de l'analyse et de constater, par exemple, que l'activité de l'UE a des incidences positives en termes de chiffres d'affaires pour les entreprises d'un secteur tout entier de l'économie du continent. C'est ce que met en évidence une étude commandée par la DGT, intitulée Study on the size of the language industry in the EU (Rinsche \& Portera-Zanotti, 2009), lorsqu'elle détaille les dépenses de l'UE pour la traduction, l'interprétation, la recherche dans le domaine des technologies du langage, etc.

Sans étudier tous les chiffres, on peut déjà voir dans le nombre d'emplois que représentent ces services de traduction un bénéfice pour la société, y compris financier: ces employés engagent nécessairement dans leur quotidien un certain nombre de dépenses, dont une partie substantielle doit en principe nourrir l'économie européenne (l'agriculture, l'industrie, les commerces, les services, etc.), puisqu'ils paient des impôts qui, à leur tour, permettent d'injecter des fonds dans cette même économie, etc. Koskinen (2008, p. 5) souligne aussi l'impact économique de la 
politique de traduction de l'UE pour le marché de la traduction finnois. Une autre étude de la Commission européenne, l'étude ELAN concernant les «incidences du manque de compétences linguistiques sur l'économie européenne», citée par Leonard Orban (2010, p. 27), ainsi que la brochure intitulée Les langues font nos affaires (Commission européenne, 2008a), montre aussi qu'une appréhension réaliste du phénomène du multilinguisme dans les entreprises, notamment par des stratégies de traduction adaptées, peut leur permettre de décrocher des marchés.

En Bretagne, alors même que l'aspect symbolique de la traduction en breton semble évident, les considérations économiques ne sont pas du tout absentes. Nous l'avons dit: la présence de la langue des ancêtres, du territoire, la «langue du cœur» (Comiti, 1992, p. 97), sur des produits peut agir comme un label de qualité, servir à orienter les choix du consommateur vers des produits régionaux, synonymes d'emplois locaux comme a voulu le montrer une campagne de publicité de l'association Produit en Bretagne, donc de ventes et d'économie... Par ailleurs, les Bretons regardent aussi les exemples gallois et irlandais où le secteur des médias en langue autochtone est source de création d'emplois. Suite aux difficultés de TV Breizh, première télévision régionale bretonne créée en 2000:

Des emplois émergents et régionalisés à Lorient (traduction notamment) ont [...] dû être supprimés (alors que, rappelonsle, la chaîne galloise $\mathrm{S} 4 \mathrm{C}$ a dans le même temps créé trois mille emplois directs et indirects). (Bretagne Prospective, 2008, p. 88)

$\mathrm{Ou}$ encore: «la relance du gaëlique, qui compte désormais 30000 locuteurs, a déjà créé en Irlande 1000 emplois peu délocalisables» (ibid., p.106). Le potentiel économique d'un secteur de la traduction en breton est donc réel, même s'il doit dans un premier temps être soutenu par les autorités publiques (v. aussi Ar Rouz, 2009). N'est-ce pas d'ailleurs le cas, indirectement, du marché finnois stimulé par l'UE?

Mais au-delà de ces considérations économiques, au demeurant fondamentales, il convient de considérer de quelle manière la traduction peut éventuellement permettre une «meilleure» communication. La question de la traduction en breton est à cet égard très intéressante 
puisqu'elle porte d'abord une revendication de la différence culturelle. Or, nous dit Gagnepain (1994, p. 42), «nous ne négocions que parce que nous avons posé des frontières». Autrement dit, en prenant acte de la différence entre les interlocuteurs, le traducteur faciliterait leur négociation; en les installant de part et d'autre d'une frontière bien définie, il leur permet un certain rapport au langage, et donc à euxmêmes et à l'Autre.

Regardons en effet, comme y invite encore Meylaerts (2009, p. 19), quelles sont les alternatives proposées à la traduction professionnelle, quel est le rôle de la non-traduction institutionnelle. François Grin, lorsqu'il met en rapport les coûts et la justice linguistique (2004), en vient à étudier de près différents régimes linguistiques possibles et met bien en évidence, après avoir relativisé le coût financier du régime "panarchique" théoriquement en vigueur, que le renoncement de certains États à l'officialité de leur langue au sein de l'UE entraînerait pour leurs locuteurs, outre une possible discrimination à l'embauche, une «fragilisation de leur position dans toute situation de débat ou de négociation (et cela, malgré un effort considérable dans l'apprentissage d'une des six langues officielles)». (Grin, 2004, p. 6)

C'est ce que soulignent également les études sur les interactions verbales: "Le LNN [locuteur non natif] étant au départ plus ou moins fortement handicapé par rapport au LN [locuteur natif], sa "face" risque de se trouver perpétuellement menacée au cours de l'échange» (Kerbrat-Orecchioni, 1995, p. 122). Et Piron en donne un exemple parlant lorsqu'il raconte:

Une ministre danoise, Mme Helle Degn, venait à peine d'entrer en fonction quand elle a dû présider une réunion internationale. S'exprimant en anglais, elle a voulu dire: «Excusez-moi, je ne connais pas bien le dossier, je viens tout juste d'entrer en fonction» et elle a dit: «I'm at the beginning of my period» (Jyllands Posten, 1994; Sprog og erbverv, 1994), ce qui veut dire: "Je suis au début de mes règles». Tout le monde a compris, mais son prestige en a pris un sacré coup. (Piron, 2001, p. 99)

Autrement dit, la traduction, en évitant aux interlocuteurs ces maladresses dues à l'expression dans une langue étrangère (à défaut de leur éviter des lapsus dans leur langue maternelle), se fait 
alors porteuse de la valeur des interlocuteurs pour eux-mêmes et dans l'échange.

Et puisque l'on parle de négociation, de préférence à la violence comme forme d'interaction, Marshall B. Rosenberg (2003), fondateur de la communication non violente (CNV), constate, de même que les formateurs dans d'autres langues (D'Ansembourg, 2004, par exemple), que la plupart des personnes rencontrées manquent cruellement de vocabulaire pour exprimer leurs émotions et leurs besoins dans leur langue maternelle. Or, c'est la base de la CNV: exprimer des faits, les émotions qu'ils provoquent, les besoins que celles-ci traduisent, ainsi que des demandes réalistes, concrètes et négociables qui permettraient à l'autre de contribuer à satisfaire ces besoins fondamentaux. Si l'on manque de vocabulaire dans sa propre langue pour mettre en place ce processus de communication, comment pourrait-on le faire si l'on doit s'exprimer dans une langue étrangère?

Grin évoque cependant d'autres modèles permettant de réduire le nombre de combinaisons de traduction: le modèle «monarchique», où une seule des langues de l'Union serait officielle, le modèle "synarchique» si cette langue est une langue tierce (par exemple le latin ou l'espéranto), le modèle «oligarchique», où l'on choisirait seulement quelques-unes des langues officielles (par exemple les langues procédurales de la Commission), le modèle «hégémonique», où toutes les langues seraient officielles mais une seule d'entre elles servirait de langue pivot pour la traduction, et enfin le modèle «technocratique», où cette langue pivot serait une langue tierce.

Mais alors, si l'on adoptait un modèle impliquant une sélection parmi les langues officielles, comment les choisiraiton? En fonction de quel critère? Si l'on considère le nombre de locuteurs dans l'Union, c'est l'allemand qui vient en premier, suivi de l'anglais et du français (voir le Tableau 1, p. 40). Si, en revanche, on décide que le nombre de locuteurs des langues dans le monde prime, on observe que l'ordre des treize premières langues n'est pas tout à fait le même (voir le Tableau 2, page suivante). On pourrait ainsi adopter différents critères, tels que la présence des langues dans les États européens (l'allemand se rencontre ainsi dans quinze États, le polonais dans neuf, le grec dans huit, etc.) et limiter cette perspective à l'officialité des langues en question dans lesdits États (l'allemand est officiel ou co-officiel, au moins sur 
une partie du territoire, dans cinq États, le français dans quatre, l'anglais dans trois, etc.). Dans tous les cas de figure envisagés ici, ce n'est jamais l'anglais qui émerge en première position. En revanche, si l'on prend en compte l'officialité des langues dans les États du monde entier, le nombre de locuteurs de langue seconde, le tableau serait différent. Si on parle de prestige et d'usage dans les grandes organisations supranationales, le choix induit serait également modifié. On observe ainsi, comme le montre Calvet, que le classement des langues les plus «importantes» change dès que l'on modifie le critère premier et qu'il ne peut donc servir à trancher en faveur de l'une ou de l'autre.

Tableau 2. Langues de l'UE selon le nombre de locuteurs dans le monde

\begin{tabular}{|l|c|}
\hline \multicolumn{1}{|c|}{ Langue (FR) } & Locuteurs dans le monde \\
\hline espagnol, castillan & 370000000 \\
\hline anglais & 321500000 \\
\hline portugais & 202000000 \\
\hline allemand & 91600000 \\
\hline français & 71400000 \\
\hline italien & 61696677 \\
\hline polonais & 39990670 \\
\hline roumain, daco-roumain & 23351080 \\
\hline néerlandais & 22500000 \\
\hline grec & 13084490 \\
\hline hongrois & 12501270 \\
\hline tchèque & 9400000 \\
\hline suédois & 9389961 \\
\hline
\end{tabular}

$\mathrm{Vu}$ l'autre aspect du coût que nous avons évoqué, la lourdeur, Grin en vient par élimination à estimer que les modèles «synarchique» et «technocratique» sont les meilleures alternatives au modèle panarchique, puisque le choix d'une des langues existantes comme langue officielle ou comme langue pivot serait trop inégalitaire pour être acceptable. Mais qu'adviendrait-il alors de cette "fragilisation» dans l'échange si tout le monde devait échanger dans une langue apprise? Si le coût d'apprentissage de l'espéranto est faible, et que cela en fait selon Grin le meilleur candidat à cette position de langue officielle unique ou de langue pivot, peut-on considérer pour autant que tous les interlocuteurs seront automatiquement à égalité dans leur rapport à cette langue 
tierce? On peut en outre se demander pourquoi l'on devrait se contenter d'une seule langue pivot. En fait, le rapport Herbillon (2003), indique que, dès 2001, le Bureau du Parlement européen avait opté pour trois langues pivots: le français, l'anglais et l'allemand. Mais est-il réellement besoin de contrôler cette langue pivot? Pourquoi ne se baserait-on pas simplement sur les compétences des traducteurs et interprètes en poste dans les institutions de l'UE?

L'incarnation de positionnements et de valeurs dans l'échange n'est pas le seul rôle de la traduction en breton, qui vise aussi explicitement à la «revalorisation» de la langue pour ses locuteurs, tout en la montrant porteuse de modernité (Ar Rouz, 2009), d'authenticité, etc. Il ne nous semble pas incongru de penser que la traduction peut jouer dans une certaine mesure un rôle similaire pour les langues officielles européennes. Si les députés et fonctionnaires européens ne parlaient que des langues officielles autres que la leur, voire une langue tierce, ne verrait-on pas les citoyens l'abandonner aussi au profit des langues valorisées dans l'institution supranationale ou, du moins, utiliser ces dernières dans différents contextes d'échanges internationaux comme la science? Et dans ce cas, qui s'alarmerait de l'appauvrissement linguistique, comme le fait aujourd'hui la British Academy au sujet de la tendance au monolinguisme des anglophones (Oustinoff et al., 2010, p. 15)?

\section{Conclusion : la traduction comme politique linguistique}

Il resterait beaucoup à dire sur la valeur de la traduction professionnelle dans des contextes plurilingues, mais on voit déjà que, si l'on prend véritablement en compte tous les éléments du bénéfice qui constitue, avec le coût, une face de l'analyse de cette valeur, le service n'apparaît plus comme un «luxe» (Calvet, 2002, p. 100 , p. 174 , p. 205). On peut l'envisager au contraire comme une étape nécessaire de la recherche d'un «bien-être ensemble» entre des pays encore déchirés par la guerre il y a soixante ans. Dans les mots (traduits) de Wagner et al. (2002, p. 26), la traduction contribue à atteindre les objectifs de l'UE que sont «la paix, la prospérité et le respect mutuel pour ses États membres». Il n'en demeure pas moins que le bénéfice de ce type de traduction est 
toujours mis en rapport à un coût, particulièrement évident et contraignant lorsqu'il s'agit de son volet économique et financier.

On observe alors que l'analyse de la valeur de la traduction professionnelle mène par exemple, dans le cas de l'UE, à une "codification" des usages en matière de rédaction (limitation de la longueur des textes originaux, aide aux rédacteurs, etc.) et de traduction (anticipation des besoins, planification, traduction à partir de textes stabilisés, dans certaines langues uniquement suivant les besoins et la fonction des documents, etc.). Ce sont là des coûts supplémentaires qui permettent de maintenir les coûts financiers à un niveau raisonnable. On peut dire à ce moment-là que le service de traduction fait partie intégrante de la politique linguistique mise en place.

Dès lors que la politique linguistique ne consiste plus véritablement, comme le préconise Calvet (2002, p. 21), à observer une situation sociolinguistique donnée, jugée insatisfaisante, et à définir les moyens d'aboutir à une situation jugée plus acceptable, mais plutôt à permettre le maintien de la situation existante, notamment en améliorant l'efficacité de la traduction professionnelle, comme la DGT a su le faire à loccasion des derniers élargissements (Vieilledent-Monfort, 2009, p. 60 et Pariente, 2010, p. 59), on peut même considérer que la traduction professionnelle constitue l'essentiel de la planification linguistique, c'est-à-dire de la mise en œuvre des décisions politiques conscientes concernant la coexistence des langues. On peut concevoir qu'elle ne soit pas applicable à toutes les situations, mais si elle fonctionne et donne satisfaction à la majorité des utilisateurs (ce qui pourrait faire l'objet d'une enquête plus détaillée), on peut s'interroger sur l'apparente nécessité de substitutions ou de restrictions linguistiques qui ressort des observations de Calvet sur l'UE.

Peut-être même une analyse plus poussée de la valeur de tels services montrerait-elle que la politique linguistique qu'ils permettent est au contraire recommandée dans bien des situations conflictuelles, y compris, voire surtout, là où les ressources semblent manquer pour la mettre en ouvre. L'exemple breton ainsi que celui des langues d'Espagne et de Grande-Bretagne dans l'Union nous montrent toutefois que la traduction professionnelle ne constitue parfois une solution acceptable que dans la mesure où ce sont les collectivités directement concernées qui en assument le coût. 


\section{Références}

AR ROUZ, David et Roseline LE SQUÈRE (2005). «Traduction et affichage public: quel(s) service(s) pour les langues régionales de Bretagne?». In C. Moïse, V. Fillol et T. Bulot, dir. Langues régionales. Marges linguistiques, $\mathrm{n}^{\circ}$ 10. Saint-Chamas, M.L.M.S. Éditeur, p. 190-206.

AR ROUZ, David (2009). «Le breton à la conquête de la modernité». In G. Nadiani, M. Giorgio Marrano et C. Rundle, dir. The Translation of Dialects in Multimedia. inTRAlinea Online Translation Journal. Special Issue. Forlì, Université de Bologne.

AR ROUZ, David (2010a). "Compte rendu». Comment l'Europe "parle" aux citoyens: un déf linguistique. Bruxelles, DLF Bruxelles-Europe. [http://www.langue-francaise.org/Bruxelles/Multi_28_09_2010_ compte_rendu.pdf].

AR ROUZ, David (2010b). «Les langues régionales: facteur de division ou manière dêtre ensemble?», Traduire, 223, $2^{\mathrm{e}}$ semestre, p. 121-140.

Association Avenir de la langue française. [http://www.avenir-languefrancaise.fr/articles.php ?lng=fr\&pg $=281]$.

BLANCHET, Philippe (2000). La linguistique de terrain. Méthode et théorie. Une approche ethno-sociolinguistique. Rennes, Presses universitaires de Rennes.

BRANCHADELL, Albert (2005). «Ahora, España ». El País, 28 juillet. [http://www.embat.com/docs/AhoraEspanaAlbertBranchadell. pdf].

Bretagne Prospective (2008). Le livre blanc de la Bretagne. Enjeux et perspectives. Nantes, Éditions du Temps.

CALVET, Louis-Jean (1996). Les politiques linguistiques. Paris, Presses universitaires de France. Coll. "Que sais-je?», n 3075.

CALVET, Louis-Jean (2002). Le marché aux langues. Essai de politologie linguistique sur la mondialisation. Paris, Plon.

CALVET, Louis-Jean (2003). «De la science en arabe à la traduction: centralité et diversité». Conférence donnée dans le cadre du colloque Quand la science parlait arabe, Le Caire.

CALVET, Louis-Jean (2007). "La mondialisation au filtre des traductions». In J. Nowicki et M. Oustinoff, dir. Traduction et mondialisation, vol. 1., Hermès, $\mathrm{n}^{\circ}$ 49. Paris, CNRS Éditions, p. 4557.

CHEVALIER, Gwendal (2008). "Gallo et breton: complémentarité ou concurrence?». In J.-P. Angoujard et F. Manzano, dir. Autour du 
gallo: état des lieux, analyses et perspectives. Cahiers de Sociolinguistique, $\mathrm{n}^{\circ}$ 12. Rennes, Presses Universitaires de Rennes, p. 75-109.

COMITI, Jean-Marie (1992). Les Corses face à leur langue. De la naissance de l'idiome à la reconnaissance de la langue. Ajaccio, Edizione Squadra di u Finusellu.

Commission européenne (s. d.). Traduction - Politiques. [http://europa. eu/languages/fr/chapter/33].

Commission européenne (2004). Promouvoir l'apprentissage des langues et la diversité linguistique. Plan d'action 2004-2006. Luxembourg, Office des publications officielles des Communautés européennes.

Commission européenne (2007). Un défi salutaire. Comment la multiplicité des langues pourrait consolider l'Europe. Propositions du Groupe des Intellectuels pour le Dialogue Interculturel. Luxembourg, Office des publications officielles des Communautés européennes.

Commission européenne (2008a). Les langues font nos affaires. Des entreprises plus performantes grâce à une connaissance accrue des langues. Recommandations du Forum des Entreprises sur le Multilinguisme établi par la Commission européenne. Luxembourg, Office des publications officielles des Communautés européennes.

Commission européenne (2008b). Parler les langues de l'Europe. Les langues dans l'Union européenne. Luxembourg, Office des publications officielles des Communautés européennes.

Commission européenne (2008c). Multilinguisme: un atout pour l'Europe et un engagement commun. COM (2008) 566. Bruxelles.

Commission européenne (2008d). Multilinguisme: un atout pour l'Europe et un engagement commun. Document d'accompagnement de la communication de la Commission au Parlement européen, au Conseil, au Comité économique et social et au Comité des régions. SEC (2008) 2443. Bruxelles.

Commission européenne (2009a). Le multilinguisme - Pour une meilleure compréhension mutuelle. Luxembourg, Office des publications officielles des Communautés européennes.

Commission européenne (2009b). Traduction et interprétation - Le sens des langues. Luxembourg, Office des publications officielles des Communautés européennes.

Commission européenne (2010). Histoire de la traduction à la Commission européenne. Luxembourg, Office des publications de l'Union européenne.

Cour des comptes (2006). "Rapport spécial $n^{\circ}$ 9/2006 relatif aux dépenses de traduction de la Commission, du Parlement européen 
et du Conseil, accompagné des réponses des institutions». Journal officiel de l'Union européenne, C 284, p. 1-39.

CRONIN, Michael (2003). Translation and Globalization. Oxon, Routledge.

CRONIN, Michael (2006). Translation and Identity. Oxon, Routledge.

D'ANSEMBOURG, Thomas (2004). Cessez d'être gentil soyez vrai. $\hat{E}$ tre avec les autres en restant soi-même. Montréal, Les Éditions de l'Homme.

DG Traduction (2007a). Multilinguisme et traduction. Luxembourg, Office des publications officielles des Communautés européennes.

DG Traduction (2007b). "Traduction: où en sommes-nous après le cinquième élargissement?» MEMO/07/76. 23 février.

DG Traduction (2009). Outils d'aide à la traduction et cycle de travail. Luxembourg, Office des publications officielles des Communautés européennes.

DLF Bruxelles-Europe (2010). «Comment l'Europe “parle” aux citoyens: un défi linguistique». Bruxelles: table ronde du 28 septembre 2010.

DOERFLINGER, Oscar (2001). «Le multilinguisme dans les institutions européennes». In R. Chaudenson, dir. L'Europe parlerat-elle anglais demain? Actes du Colloque international de Bordeaux organisé par le Goethe Institut et l'INTIF (3 mars 2001). Paris, Institut de la Francophonie, L'Harmattan, p. 135-138.

European Bureau For Lesser-Used Languages (2009). «Welsh takes its place at the European Commission». 22 juillet 2009.

GAGNEPAIN, Jean (1994). Leçons d'introduction à la théorie de la médiation. Anthropo-logiques, 5. Louvain-la-Neuve, Peeters.

GRIN,François (2004). «Coûts et justice linguistique dans l'élargissement de l'Union européenne ». In M. Favre d'Echallens, dir. L'avenir s'écrit aussi en français. Panoramiques, $\mathrm{n}^{\circ}$ 69. $4^{\mathrm{e}}$ trimestre, p. 97-104.

HERBILLON, Michel (2003). Rapport d'information sur la diversité linguistique dans l'Union européenne. n’ 902.11 juin. Paris, Assemblée nationale.

HICKS, Davyth (2009). «Scottish Government signs a formal agreement for the use of Gaelic in the EU». 12 octobre.

KERBRAT-ORECCHIONI, Catherine (1995, [1990]. Les interactions verbales. Tome 1. Paris, Armand Colin, coll. «Linguistique».

KOSKINEN, Kaisa (2008). Translating Institutions. An Ethnographic Study of EU Translation. Manchester, St. Jerome Publishing.

LE BOËTTÉ, Isabelle (2003). «Langue bretonne et autres langues: pratique et transmission». Octant, $\mathrm{n}^{\circ}$ 92, janvier, p. 18-22. 
Linguamon casa de les llengües. [http://www10.gencat.net/casa_ llengues/AppJava/fr/index.jsp].

MEYLAERTS, Reine (2009). «Et pour les Flamands, la même chose: quelle politique de traduction pour quelles minorités linguistiques?». Meta, 54, 1, p. 7-21.

NOWICKI, Joanna et Michaël OUSTINOFF, dir. (2007). Traduction et mondialisation, vol. 1., Hermès, $\mathrm{n}^{\circ}$ 49, p. 45-57.

NOWICKI, Joanna, Michaël OUSTINOFF et Serge PROULX, dir. (2008). L'épreuve de la diversité culturelle., Hermès, $\mathrm{n}^{\circ}$ 51. Paris, CNRS Éditions.

Office de la langue bretonne/Ofis ar brezhoneg (2006). Rapport d'activité 2005. Rennes.

ORBAN, Leonard (2010). "Langues et traduction: une politique cruciale pour l'Union européenne». In M. Oustinoff, J. Nowicki et J. Machado Da Silva, dir. Traduction et mondialisation, vol. 2., Hermès, n 56. Paris, CNRS Éditions, p. 23-28.

OUSTINOFF, Michaël, Joanna NOWICKI et Juremir MACHADO DA SILVA, dir. (2010). Traduction et mondialisation, vol. 2., Hermès, $\mathrm{n}^{\circ}$ 56. Paris, CNRS Éditions.

PARIENTE, Audrey (2010). Histoire de la traduction à la Commission européenne. Luxembourg, Office des publications de l'Union européenne.

Parlement européen (2008). Code de conduite du multilinguisme. [http:// www.europarl.europa.eu/pdf/multilinguisme/code_conduct_ multilingualism_fr.pdf].

Parlement européen (2010). «Adoption définitive du budget général de l'Union européenne pour l'exercice 2010 ». Journal officiel de l'Union européenne, $\mathrm{n}^{\circ} \mathrm{L} 064.12$ mars. .

PEETERS, Jean (1999). La médiation de l'étranger. Une sociolinguistique de la traduction. Arras, Artois Presses Université.

PIRON, Claude (2001). «L'Européen trilingue: un espoir réaliste?». In R. Chaudenson, dir. L'Europe parlera-t-elle anglais demain? Actes du Colloque international de Bordeaux organisé par le Goethe Institut et l'INTIF (3 mars 2001). Paris, Institut de la Francophonie, L'Harmattan, p. 93-102.

Région Bretagne (2004). Une politique linguistique pour la Bretagne. $7^{\mathrm{e}}$ réunion. Décembre. [http://www.bretagne.fr/internet/upload/ docs/application/pdf/2008-12/plan_de_politique_lingusitique. pdf]. 
Région Bretagne (2010). «Un nouvel Etablissement public de coopération culturelle pour la langue bretonne». [http://www.bretagne.fr/ internet/jcms/preprod_86387/un-nouvel-etablissement-public-decooperation-culturelle-pour-la-langue-bretonne].

RINSCHE, Adriane et Nadia PORTERA-ZANOTTI (2009). Study on the Size of the Language Industry in the EU. Luxembourg, European Commission.

ROSENBERG, Marshall B. (2003 [1999]). Nonviolent Communication. $A$ Language of Life. Encinitas, PuddleDancer Press.

Summer Institute Of Linguistics (SIL INTERNATIONAL). [http:// www.ethnologue.com].

UNESCO, Index translationum. [http://portal.unesco.org/culture/fr/ ev.php-url_id=7810\&url_do=do_topic\&url_section=201.html].

VIEILLEDENT-MONFORT, Catherine (2009). Études sur la traduction et le multilinguisme. La traduction à la Commission: 19582010. Bruxelles, Commission européenne. [http://ec.europa.eu/dgs/ translation/publications/index_fr.htm].

WAGNER, Emma, Svend BECH et Jesús M. MARTÍNEZ (2002). Translating for the European Union Institutions. Manchester, St. Jerome Publishing.

David ar Rouz

Université de Haute-Bretagne (Rennes 2)

LIDILE

Campus Villejean

Place du recteur Henri Le Moal

CS 24307

35043 RENNES CEDEX

FRANCE

david.leroux@univ-rennes2.fr 